\section{OPEN ACCESS}

Edited by:

Ivona Aksentijevich,

National Human Genome Research Institute (NHGRI), United States

Reviewed by:

Francesca Capon, King's College London,

United Kingdom

Giulio Cavalli,

Vita-Salute San Raffaele

University, Italy

*Correspondence: Wang-Dong Xu loutch123@163.com

An-Fang Huang

loutch211@163.com

Lin-Chong Su

linchong2019@163.com

Specialty section:

This article was submitted to Autoimmune and Autoinflammatory

Disorders,

a section of the journal

Frontiers in Immunology

Received: 23 April 2019 Accepted: 11 October 2019 Published: 31 October 2019

Citation:

Yuan Z-C, Xu W-D, Liu X-Y, LiU X-Y, Huang A-F and Su L-C (2019) Biology

of IL-36 Signaling and Its Role in

Systemic Inflammatory Diseases.

Front. Immunol. 10:2532.

doi: 10.3389/fimmu.2019.02532

\title{
Biology of IL-36 Signaling and Its Role in Systemic Inflammatory Diseases
}

\section{Zhi-Chao Yuan ${ }^{1}$, Wang-Dong Xu ${ }^{1 *}$, Xiao-Yan Liu ${ }^{2}$, Xing-You Liu ${ }^{3}$, An-Fang Huang ${ }^{4 *}$ and Lin-Chong Su ${ }^{5 *}$}

${ }^{1}$ Department of Evidence-Based Medicine, School of Public Health, Southwest Medical University, Luzhou, China, ${ }^{2}$ Department of Nutrition and Food Hygiene, School of Public Health, Southwest Medical University, Luzhou, China, ${ }^{3}$ School of Traditional Chinese Medicine, Southwest Medical University, Luzhou, China, ${ }^{4}$ Department of Rheumatology and Immunology, Affiliated Hospital of Southwest Medical University, Luzhou, China, ${ }^{5}$ Department of Rheumatology and Immunology, Minda Hospital of Hubei Minzu University, Enshi, China

Interleukin (IL)-36 is a member of the IL-1 superfamily and includes three agonists

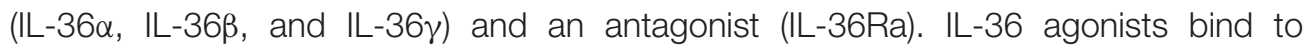
heterodimeric receptor complexes. Then, the heterotrimer complexes signal via intracellular functional domains, binding to downstream signaling proteins and inducing inflammatory responses. In this review, we summarized the current knowledge about the biological role of IL-36 and its correlation with systemic inflammatory diseases. The information collected will help to increase the understanding of the potential of IL-36 and may give clues for developing novel therapeutic strategies.

Keywords: IL-36, autoimmunity, immune cell, inflammation, cytokine

\section{INTRODUCTION}

Interleukin (IL)-36 is an inflammatory cytokine and is a member of the IL-1 superfamily. IL-36

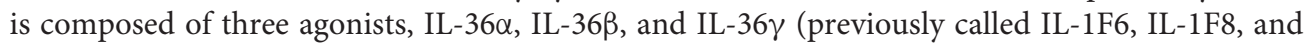
IL-1F9) and an antagonist, IL-36 receptor antagonist (IL-36Ra, formerly known as IL-1F5). This terminology was unified into its current name in 2010 (1). The genes that encode for the IL-36 family of proteins are located on human chromosome $2(2,3)$. IL-36Ra is encoded by gene IL-36RN. IL-36Ra and IL-1Ra have 52\% homologous amino acid sequence, and both function as receptor antagonists. IL-36 proteins are widely expressed in T cells, keratinocytes, and skin, lung, and gut cells. IL-36 agonists bind to receptors [IL-36R and IL-1 receptor accessory protein (IL-1RAcP)] and then activate the adaptor protein myeloid differentiated protein 88 (MyD88), mitogen-activated protein kinase (MAPK), and nuclear factor-kappa B (NF-кB) signaling pathways. Finally, these pathways initiate the regulation of target genes (4) (Figure 1). IL-36 is involved in immune cell activation, antigen presentation, and pro-inflammatory factor production. In recent years, IL-36 has attracted great interest because of its dysregulation in inflammatory diseases. The present study summarized information about the biology of IL-36 and the importance of IL-36 in common and polygenic inflammatory disorders, such as psoriasis, systemic lupus erythematosus (SLE), rheumatoid arthritis (RA), and inflammatory bowel disease (IBD). 


\section{Processing of IL-36}

IL-36 cytokines are inactive as full-length proteins and require post-translational processing to unleash their proinflammatory activity. Evidence has suggested that neutrophilderived cathepsin $G$ (Cat $G$ ), elastase, and proteinase-3 affect the processing and activation of IL-36 $\alpha$, IL-36 $\beta$, and IL$36 \gamma$. Incubation of the three IL-36 cytokines with activated neutrophil supernatants results in robust activation of these proteins, suggesting that soluble neutrophil proteases released during degranulation can activate IL-36 cytokines (5). For instance, incubation with either Cat $G$ or elastase induces IL$36 \alpha$ processing and activation by cleavage at lysine 3 (Lys3) and alanine 4 (Ala4), respectively (6). IL-36 $\beta$ was selectively stimulated by $C$ at $G$ through its cleavage at residue arginine 5 (Arg5). IL-36 $\gamma$ was robustly activated by elastase or proteinase3 by means of cleavage at the residue valine 15 (Val15) (6). In human primary dermal fibroblasts, keratinocytes, and skin equivalents, IL-36Ra is cleaved into an active form by neutrophilderived protease elastase (7). Cathepsin S (Cat S) was the major IL-36 $\gamma$-activating protease expressed by barrier tissues, and Cat $S$ functions by cleaving IL-36 $\gamma$ between residues glutamic acid 17 (Glut17) and serine 18 (Ser18) (8). The processing of IL-36 is similar to that of other members of the IL- 1 family, such as IL-37 and IL-1 $\beta$. For example, intracellular pro-IL-37 is activated by caspase-1, and mature IL-37 translocates into the nucleus to inhibit the transcription of pro-inflammatory genes. Extracellular pro-IL-37 was affected by proteases to play an anti-inflammatory role (9). Pro-IL-1 $\beta$ undergoes proteolytic cleavage by caspase- 1 to be processed into its active form, pro-inflammatory cytokine IL-1 $\beta$ (10).

\section{Biological Functions of IL-36}

IL-36 plays a role in different cell types as shown in human and animal models via binding to IL-36R. IL-36R transcripts cannot be detected in $\mathrm{CD}^{+}$or $\mathrm{CD}^{+} \mathrm{T}$ cells or in neutrophils isolated from normal mouse blood (11). Monocytes, myeloid dendritic cells (mDCs), and monocyte-derived dendritic cells (MDDCs) from normal mice expressed IL-36R and responded to IL-36, and the mDCs' surface expressed more IL-36R than that expressed on monocytes. In skin keratinocytes of normal mice and human, IL-36R was abundantly expressed (11). The role of IL-36 in keratinocytes has been widely discussed in recent years. Cat Gprocessed IL-36 $\beta$ is expressed in primary human keratinocytes. The active IL-36 $\beta$ induced the expression of inflammatory cytokines and chemokines from keratinocytes, including IL-17C, granulocyte colony-stimulating factor (G-CSF), IL-8, chemokine (C-X-C motif) ligand 1 (CXCL-1), and chemokine (C-C motif) ligand 20 (CCL-20) (6). IL-36 $\beta$ also induced IL-17A and tumor necrosis factor (TNF) expression in human keratinocytes, which could be synergized by IL-22 (12). Interestingly, IL-36 synergized with IL-17A and TNF- $\alpha$ to increase the expression of IL6, IL-8, and TNF- $\alpha$ in primary human keratinocytes (13). Normal human keratinocytes stimulated with IL-36 $\alpha$, IL-36 $\beta$, or IL-36 $\gamma$ produced increased levels of CCL3, CCL4, CCL20, CCL5, CXCL8, and CCL20, demonstrating that, following IL36 exposure, keratinocytes are potent sources of macrophage, $\mathrm{T}$ cell, and neutrophil chemokines (11). When keratinocytes from healthy donors were cultured with IL-36 $\alpha$, IL-36 $\beta$, or IL$36 \gamma$, the expression of IL-17A signaling-related genes (IL36G, S100A7, and LCN2), p38-MAPK signaling-related genes (IRAK2 and PLA2G4D), and granulocyte/agranulocyte adhesion and diapedesis [CCL20, IL-8, and matrix metallopeptidase 9 (MMP9)] were upregulated, suggesting that IL-36 cytokines are able to amplify keratinocyte inflammatory responses by promoting not only their own expression but also that of molecules related to Th17 signaling (14). Levels of genes encoding interferon (IFN) receptor components, such as IFN gamma receptor 1 (IFNGR1), IFNGR2, and IFNAR2, were increased by IL-36 (IL-36 $\alpha$, IL-

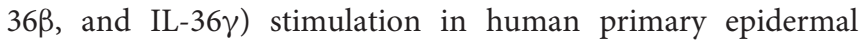
keratinocytes (15). Interestingly, the expression of genes with two or more IFN regulatory factor 1 (IRF1) binding sites

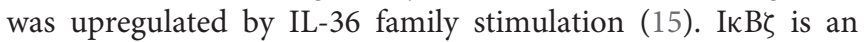
atypical I $\mathrm{B}$ (inhibitor of NF- $\kappa \mathrm{B}$ ) member, and it is a specific transcriptional regulator for $N F-\kappa B$ target genes. I $\kappa \mathrm{B} \zeta$ expression was induced when human skin epidermal keratinocytes were exposed to IL-36 (16). It is notable that IL-36-mediated induction of $\mathrm{I} \kappa \mathrm{B} \zeta$ was required for the expression of downstream genes involved in inflammatory signaling, neutrophil chemotaxis, and leukocyte activation (16). In addition, IL-36 $\gamma$-stimulated human endothelial cells promoted the generation of IL-8, CCL2, CCL20, and adhesion molecules [vascular cell adhesion molecule-1 (VCAM-1) and intercellular adhesion molecule (ICAM-1)] was upregulated in IL-36 $\gamma$-treated endothelial cells (17). Therefore, IL-36 may regulate keratinocyte- and endothelial cell-mediated inflammatory response.

Human monocytes cultured with IL-36 were activated, and IL36 stimulation significantly upregulated expression of IL- $1 \alpha$, IL$1 \beta$, and IL-6 (11). In murine dendritic cells (DCs), IL-36 agonist treatment upregulated activation markers of DCs, such as CD80, CD86, and MHCII, and it induced the production of IL- 6 and IL-12 (18). When murine MDDCs were stimulated with IL-36 $\beta$, the levels of IL-12p70, IL-23, and IL-10 became elevated (19). Furthermore, in IL-36 $\alpha$ knockout (-/-) mice, the number of neutrophils recruited to the epidermis and dermis was reduced, and CXCL1 generation was downregulated (20). Together, the above findings indicate that IL-36 plays an important role in innate immune response.

When $\mathrm{CD}^{+} \mathrm{T}$ cells from $\mathrm{IL}-36 \mathrm{R}^{-/-}$mice were cocultured with IL-36 $\gamma$, regulatory $\mathrm{T}$ (Treg) cell differentiation was inhibited compared with $\mathrm{CD}^{+} \mathrm{T}$ cells from wild-type

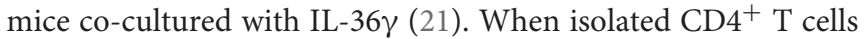

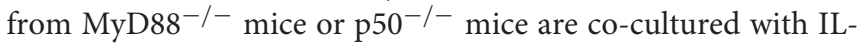
$36 \gamma$, the differentiation of Treg cells increased (21). In contrast, abrogation of IL-36 $\gamma$-induced IL-9 production was observed in $\mathrm{CD} 4{ }^{+} \mathrm{T}$ cells from $\mathrm{MyD} 88^{-/-}$or $\mathrm{p} 50^{-/-}$mice when stimulated

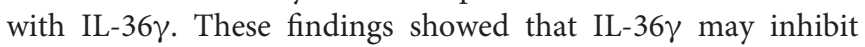
Treg cell differentiation and promote Th9 cell differentiation by downstream signaling pathways, including MyD88 and NF-кB (21). CD ${ }^{+}$T cells stimulated with IL-36 $\alpha$ under Th1 polarizing conditions showed that IL-36 $\alpha$ potently drove Th1 responses (22). IL-36 $\beta$ upregulates the production of IL-18 and IL-12p70 in MDDCs, suggesting the induction of a Th1 phenotype (23). These studies illustrated that IL-36 is important in effector T-cell differentiation (Figure 2). 


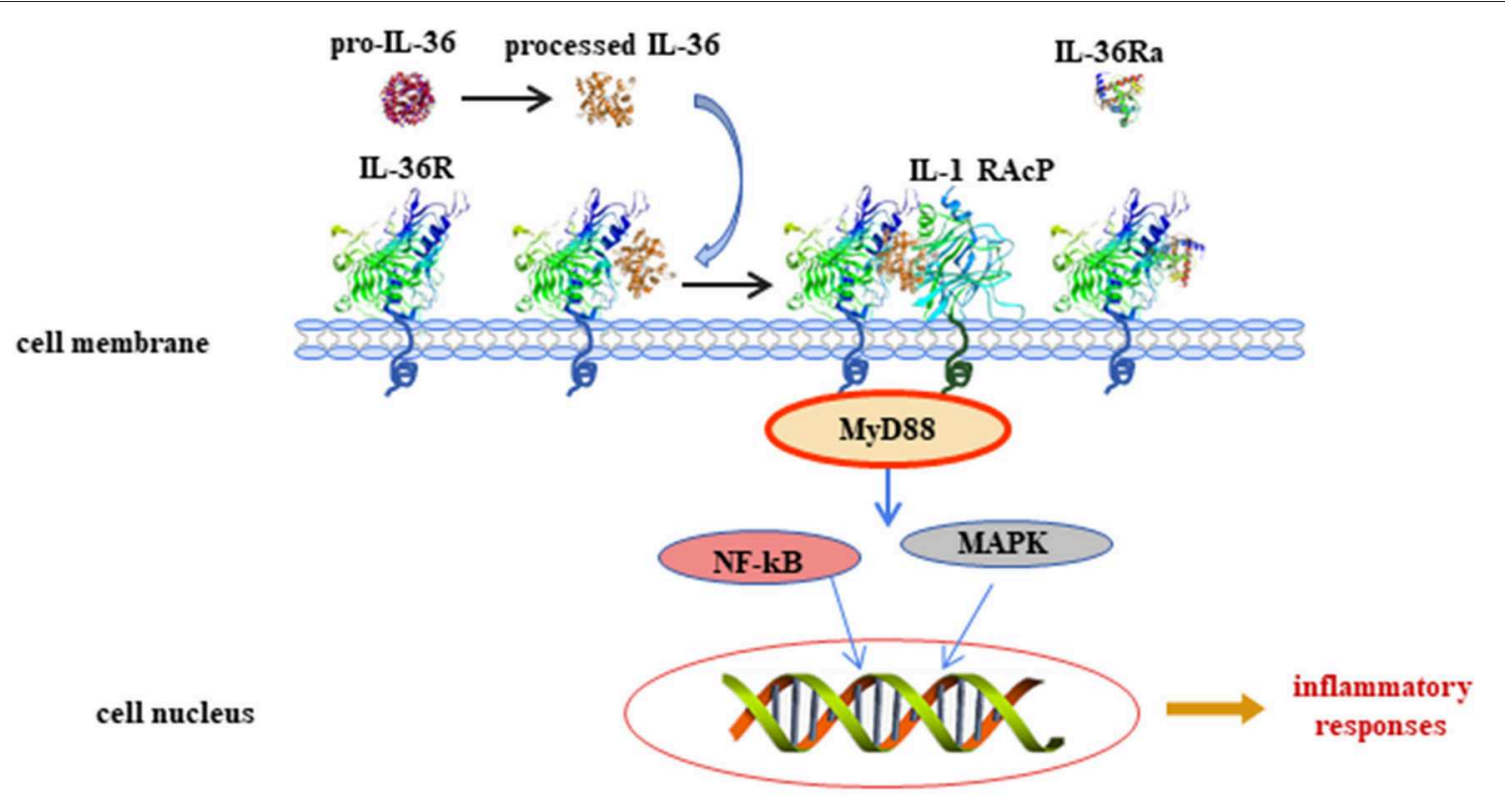

FIGURE 1 | Interleukin (IL)-36 signal pathways induce inflammatory responses. Pro-IL-36 cytokines are inactive proteins and require post-translational processing to fully unleash the pro-inflammatory activity. IL-36 agonists bind to heterodimeric receptor complexes, including IL-36 receptor (IL-36R) and co-receptor IL-1 receptor accessory protein (IL-1RAcP). Subsequently, the heterotrimer complex binds to adaptor protein myeloid differentiated protein 88 (MyD88), activating

mitogen-activated protein kinase (MAPK) and nuclear transcription factor kappa B (NF- $\kappa$ B) signaling cascade pathways, and regulates expression of target genes.

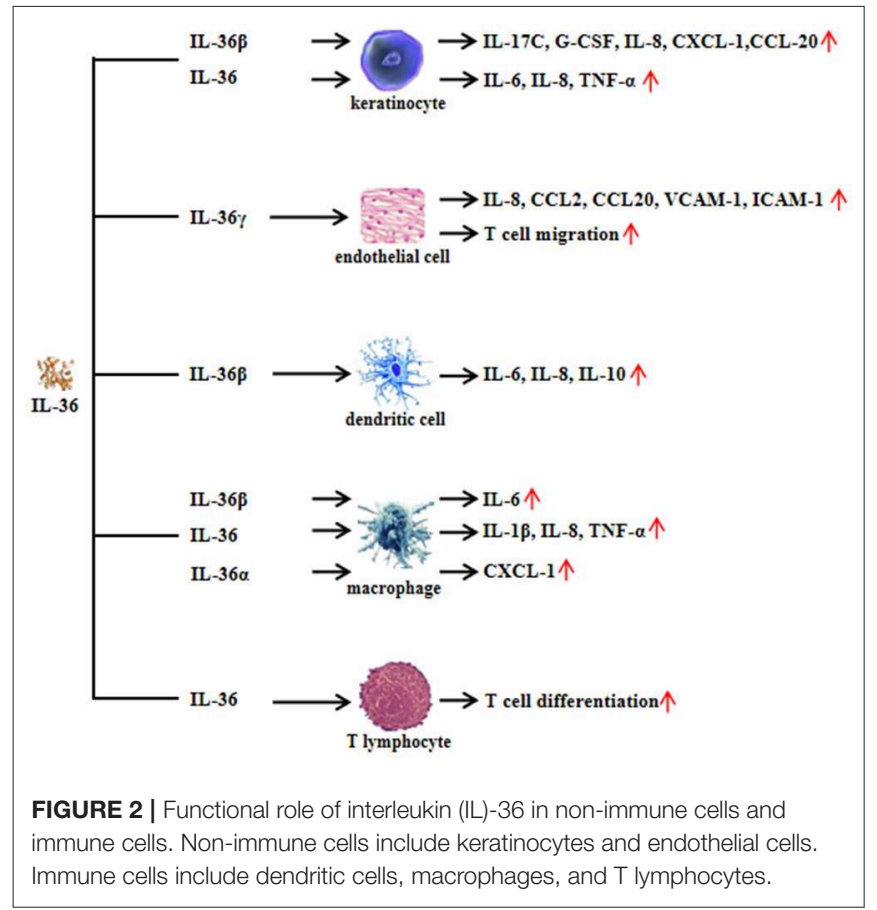

\section{Regulation of IL-36}

Available evidence has suggested that IL-36 regulates the function of both non-immune cells and immune cells. IL-36 can also be regulated by different inflammatory components and cells. In mouse keratinocytes, IL-1 $\alpha$ induced IL-36 $\alpha$ expression, and the levels of IL-36 $\alpha$ from inflamed IL-1R1 $1^{-/-}$skin was significantly lower than those of wild-type mice (11). Therefore, IL-1 $\alpha$ is an important regulator of IL-36 $\alpha$ expression. In return, IL$36 \alpha$ may regulate IL- $1 \alpha$ in a feedback loop, where primary mice keratinocytes rapidly induced IL- $1 \alpha$ in response to IL$36 \alpha$ stimulation. Interestingly, the induction of IL-1 $\alpha$ correlated with increased IL-36 $\alpha$ expression (11). Moreover, the levels of IL- $1 \alpha$ released from imiquimod-treated skin were significantly lower in the absence of IL-36 $\alpha$ than in the presence of IL-36 $\alpha$ (11). Therefore, this result suggested that IL-36 $\alpha$ may induce IL-1 $\alpha$ expression. In human keratinocytes, IL-22, IL-17A, and TNF- $\alpha$ induce the production of all three IL-36 subfamilies, and IFN- $\gamma$ selectively induces IL-36 $\beta$ production (13). With macrophage-activating lipopeptide 2 (MALP-2) stimulation, IL-36 $\alpha$ expression was highly enhanced in human primary keratinocytes (24). The double-stranded RNA analog poly(I:C) induces pyroptosis in human keratinocytes, thereby facilitating the extracellular release of IL-36 $\gamma$, whereas suppression of caspase-3/7 and caspase-1 blocks the release of IL-36 $\gamma$ from poly(I:C)-treated cells (25). IL-38 is known to function as an antagonist of IL-36R (26). IL-38 binds to IL-36R and IL-1RAcP, inhibiting the biologic function of IL-36 (19) (Figure 3).

\section{Role of IL-36 in Systemic and Inflammatory Diseases \\ IL-36 in SLE}

SLE is an autoimmune disease characterized by damage to multiple systems and organs (such as kidney, bones, and skin) and dysregulated autoantibody production. Studies have shown 


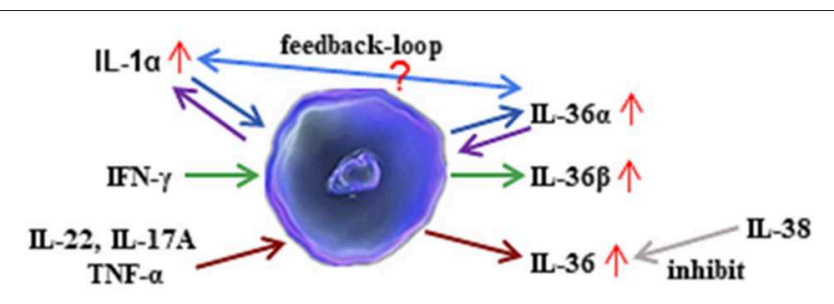

keratinocyte

FIGURE 3 | Regulation of interleukin (IL)-36 in keratinocytes. IL-36 $\alpha$ regulates the generation of $\mathrm{IL}-1 \alpha$ in keratinocytes. In turn, $\mathrm{IL}-1 \alpha$ affects the synthesis of IL-36 $\alpha$ in keratinocytes. However, the mechanism of feedback loop needs to be elucidated in the future. Interferon (IFN)- $\gamma$ induces IL-36 $\beta$ production in keratinocytes. In addition, inflammatory cytokines such as IL-22, IL-17A, and tumor necrosis factor (TNF)- $\alpha$ induce all three agonists' expression, whereas the effect may be inhibited by IL-38 administration.

that IL-36 expression and function may correlate with the pathogenesis of SLE. In the serum and tubuli of nephritic kidney biopsies of SLE patients, IL-36 expression (IL-36 $\alpha$ and IL-36 $\gamma$ ) was significantly higher than that in healthy controls (27). Elevated expression of IL-36 $\alpha$ and IL-36 $\gamma$ positively correlated with the SLE disease activity index. In contrast, serum levels of IL-36Ra were significantly lower in SLE patients than in healthy controls (28-30). IL-36 stimulation promoted the production of inflammatory cytokines that participate in the development of SLE, including IFN- $\gamma$, TNF- $\alpha$, IL-4, IL17, IL-6, and IL-8 (30). Evidence suggested that regulatory $\mathrm{B}$ cell (Breg) subsets, such as $\mathrm{CD} 19^{+} \mathrm{CD} 24^{\text {high }} \mathrm{CD} 27^{+}$Bregs, could downregulate adaptive/innate immune responses (28), and the proportion of $\mathrm{CD} 19^{+} \mathrm{CD} 24^{\text {high }} \mathrm{CD} 27^{+}$cells in total $\mathrm{B}$ lymphocytes negatively correlated with the proportion of IL36R-positive B lymphocytes. It is hypothesized that there may be an inverse correlation between circulating inflammatory IL36 and anti-inflammatory Breg subpopulations. However, how IL-36 regulates this specific subset and, therefore, contributes to SLE pathogenesis needs further investigation. In addition, in our previous studies, Th17 cell-related cytokines, such as IL-17 and IL-21, have been found to contribute to SLE pathogenesis $(31,32)$. Interestingly, IL-17 $-/-$ mice did not develop lupus after pristane injection, whereas wild-type mice developed lupus (33), as evidenced by increased autoantibody production and the development of glomerulonephritis. These data showed that Th17/IL-17 is important in lupus pathogenesis (33). Since IL36 is able to increase the expression of IL-17 in lupus, what is the role of IL-36 in the regulation of Th17/IL-17 signaling? How does IL-36 interact with IL-17 and, therefore, contribute to the development of lupus? Does IL-36 regulate some downstream signaling pathways and then affect the production of IL-17, aggravating lupus development? Hopefully, all these questions will be answered in the future.

\section{IL-36 in Psoriasis}

Psoriasis is a chronic and relapsing inflammatory skin disease. Plaque psoriasis is the most common form of the disease with a worldwide prevalence 1-2\%. The main pathological manifestations include excessive epidermal keratinization and parakeratosis, hypertrophy of stratum spinosum cells, lymphocyte infiltration, and vasodilation. Genetic factors, infections, and immune cell dysfunction play important roles in this complex and polygenic autoimmune disease. Epidemiological studies involving twins and families suggest that psoriasis is an inheritable disease. For instance, monozygotic twins had higher concordance of psoriasis than dizygotic twins (34). Variation in the $H L A-C$ gene contributes to psoriatic heredity, by which HLA-C*06 is related to psoriasis patients in White (35) and Chinese (36) populations. A genome-wide association study identified numerous genetic risk factors associated with psoriasis, such as LCE3B, LCE3C, CSTA (involving skin barrier function), IL12B, IL23A, IL23R, TYK2, $I F I H 1$, and ERAP1, and ZAP70 (in immunological response) (37). Another study with whole-exome single-nucleotide polymorphism array found that genes such as C1orf141, ZNF683, TMC6, AIM2, IL1RL1, CASR, SON, ZFYVE16, and MTHFR were related to psoriasis in Chinese (38). These findings indicated that psoriasis is a polygenic disorder. As compared to psoriasis, deficiency of IL-36R antagonist (DITRA) is a life-threatening monogenic type of disease caused by loss-of-function mutations in the IL36RN gene. Affected patients suffered from recurrent episodes of generalized pustular psoriasis with skin impairment and systemic inflammation. Several pathogenic variants in the $I L-36 R N$ gene have been recognized to correlate with DITRA, such as c.41C >A, c.41T $>$ C, and c.420_426del (39). Clinically, patients with DITRA present with symptoms in their childhood or adolescence. Inhibition of TNF- $\alpha$, IL-12/23, and IL-17 is effective in suppressing the disease activity in patients with DITRA, while anti-IL-1 treatment seems less effective (40). Therefore, DITRA is an example of monogenic IL-36-mediated disease, which is different from psoriasis.

Serum levels of IL-36 in patients with psoriasis were higher than those in healthy controls, and the elevated levels of IL36 correlated with disease activity $(41,42)$. A recent study that discussed anti-IL-36 treatment in pustular psoriasis patients showed that the monoclonal antibody against IL-36R reduced symptoms of the disease (43). In imiquimod-induced psoriasis (a psoriasis mouse model), IL-36 $\alpha$ injection contributed to the development of psoriasis, especially severe skin lesions (20). Psoriatic mice treated with IL-36R-blocking antibodies showed improved psoriatic dermatitis (14). IL-36 signalingrelated genes were upregulated in psoriatic skin lesions and enriched within psoriasis susceptibility loci when human primary keratinocytes were treated with bioactive IL-36 (14). With IL-

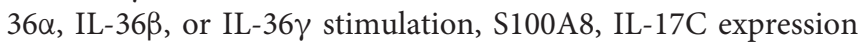
is increased in wild-type keratinocytes compared to IL-36 $\mathrm{R}^{-/-}$ keratinocytes (44), suggesting the role of the IL-36/IL-36R autocrine loop within keratinocytes in psoriasis development. AP1S3 mutations have been widely demonstrated as risk factors in psoriasis. AP1S3 deficiency enhanced Toll-like receptor (TLR) 2/6 signaling, such as MALP-2, which induced the expression of IL-36 $\alpha$ in keratinocytes and impaired keratinocyte autophagy. Furthermore, individuals with psoriasis showed a particularly severe, recalcitrant phenotype that carried both the IL36RN and 
AP1S3 mutations (24). IL-36 combined with IL-1 to recruit neutrophils in the dermis and epidermis and, then, promoted the inflammatory keratinocyte response via inducing the expression of inflammatory chemokines, such as IL-8 (45), suggesting that the IL-36/IL-1-chemokine-neutrophil axis may have a role in the pathogenesis of psoriasis. In addition, keratinocytes stimulated with IL-36 (IL-36 $\alpha$, IL-36 $\beta$, and IL-36 $\gamma$ ) significantly upregulated CCL20, MMP9, and IRAK2 (14). It has been shown that Th17 cells and related cytokines (IL-23/IL-17/IL-22 axis) participated in the pathogenesis of psoriasis (46). In imiquimod-induced psoriasis mice, IL-36Ra deficiency drove skin lesions and induced IL-23, IL-17, and IL-22 expression, whereas the wild-type mice did not develop skin lesions (46). These findings suggested that IL-36 contributes to the pathogenesis of psoriasis.

\section{IL-36 in Arthritis}

$\mathrm{RA}$ is a chronic inflammatory autoimmune disease. The pathological symptom of RA is synovitis of the joints, with the proliferation of synovial cells, the formation of neovascularization, and local infiltration of lymphocytes. IL$36 \alpha$, IL-36R, and IL-36Ra were detected in the synovial tissues of patients with RA, and the expression of IL-36 $\alpha$ in the synovial tissue was higher in RA patients than in osteoarthritis (OA) patients (47). IL-36 stimulation with synovial fibroblasts promoted the production of IL-6 and IL-8 (47). Similarly, IL$36 \mathrm{R}$ and IL-36 $\gamma$ were highly expressed in the joints of collageninduced arthritis mice (48). The limited data indicated that IL-36 may correlate with RA pathogenesis. Spondyloarthritides (SpA) are a group of chronic systemic inflammatory joint diseases, and psoriatic arthritis (PsA) is one of the main types. The expression of IL-36R and its ligands IL-36 $\alpha$ and IL-36Ra can be detected in the synovial lining layer and in the cellular infiltrates of patients with PsA (47). IL-36 $\alpha$ expression was higher in PsA patients compared with that in OA patients. $\mathrm{CD}_{138^{+}}$plasma cells were the main cellular source of IL-36 $\alpha$, which promoted the generation of IL- 6 and IL-8 in fibroblast-like synoviocytes by activating the $\mathrm{p} 38 / \mathrm{NF}-\kappa \mathrm{B}$ signaling pathways (47). OA is a debilitating disease, and older people are more susceptible to this common disorder. IL-36 $\alpha / \mathrm{IL}-36 \mathrm{R}$ expression was increased in OA patients' cartilage compared to controls (49). Mice deficient in TGFBR2 showed an uncavitated, disorganized cluster of cells and abnormal condyle morphology after the destabilization of the medial meniscus (DMM) procedure, whereas wild-type mice developed knee joint cavities containing cartilage, menisci, and supporting ligaments in the knees (49). Similarly, after DMM surgery, wild-type mice showed a decrease in IL-36Ra and an increase in IL-36 $\alpha$, IL-36 $\beta$, IL-36 $\gamma$, and IL-36R within the regions of increased mechanical loading, indicating that, in articular cartilage, the abnormal mechanical loading caused by DMM may trigger downregulation of IL-36Ra and upregulation of IL-36/IL$36 \mathrm{R}$ to induce OA progression (49). Moreover, after injection of IL-36Ra into the knee joints of TGFBR2-/- mice or DMMinduced $\mathrm{OA}$ mice, there was attenuation of OA progression, accompanied with a decrease in chondrocyte hypertrophy, reduced fibrillation, reduced matrix destruction, and reduced expression of collagen 10 and MMP13. It is notable that the injection of IL-36 $\alpha$ not only induced an OA-like phenotype in wild-type mice but also worsened OA in TGFBR2 ${ }^{-/-}$mice, suggesting that IL-36 $\alpha$ promoted OA development (49).

\section{IL-36 in IBD}

IBD is a chronic intestinal inflammatory disease that encompasses Crohn's disease and ulcerative colitis. The causes of the disease remain unknown. IL-36 $\alpha$ and IL-36 $\gamma$ were elevated in mucosal biopsies from patients with $\operatorname{IBD}(50,51)$. In dextran sodium sulfate (DSS)-induced colitis mice, IL-36 administration promoted colon injury and inflammation, and the colonic expression of IL-36 $\alpha$ and IL-36 $\gamma$ was markedly elevated. Interestingly, IL-36 $\mathrm{R}^{-/-}$mice showed reduced disease progression after treatment with DSS, whereas wild-type mice developed severe symptoms in the presence of DSS (22). CD4 ${ }^{+}$ $\mathrm{T}$ cells from the mucosa of the colon of mice with colitis treated with IL-36 $\alpha$ and IL-36 $\gamma$ showed significant induction of Th1 cell differentiation (22). Furthermore, IL-36 $\alpha$ and IL-36 $\gamma$ stimulation induced the expression of chemokines and acute phase proteins in an intestinal epithelial cell line (HT-29 cells) and induced the assembly of MyD88, TRAF6, IRAK1, and TAK1 and the transactivation of NF- $\kappa \mathrm{B}, \mathrm{AP}-1$, and MAPK, therefore enhancing inflammation of the colon (52). The above studies showed that IL-36 is involved in the pathogenesis of IBD.

\section{IL-36 in Other Systemic Inflammatory Diseases}

Studies indicated that IL-36 family cytokines have emerged as important inflammatory mediators in different inflammatory autoimmune disorders, such as SpA and primary Sjögren's syndrome (pSS). SpA comprises a group of diseases including ankylosing spondylitis (AS), reactive arthritis (ReA), PsA, and IBD-related arthropathy (53). IL-38 gene polymorphisms are associated with both AS and PsA $(54,55)$. Interestingly, IL38 and IL-36Ra have demonstrated similar anti-inflammatory effects. Previous studies have reported that the pathogenesis of psoriasis is related to the IL-23/IL-17/IL-22 axis. Therefore, it is hypothesized that IL-36 may be involved in the pathogenesis of SpA by increasing the generation of pro-inflammatory cytokines, such as IL-23, IL-17, IL-22, TNF- $\alpha$, and IL-6, as well as promoting the inflammatory response, similar to the role of IL-38 in SpA. However, further studies are needed to validate this hypothesis. Serum levels of IL-36 $\alpha$ were elevated in pSS patients compared with those in healthy controls (56). The expression of IL-36 $\alpha$ in the minor salivary glands of pSS patients was significantly

TABLE 1 | Abnormal expression of interleukin (IL)-36 in inflammatory diseases.

\begin{tabular}{llc}
\hline Disorder & Expression of IL-36 & References \\
\hline Systemic lupus erythematosus & Serum & $(27-30) \uparrow$ \\
Psoriasis & Serum & $(41,42) \uparrow$ \\
Rheumatoid arthritis & Synovial tissue & $(42,47) \uparrow$ \\
Inflammatory bowel disease & Colonic mucosa & $(50,51) \uparrow$ \\
Primary Sjögren's syndrome & Serum & $(57) \uparrow$ \\
Blistering disease & Serum & $(56) \uparrow$
\end{tabular}


higher compared to that in controls. It is notable that $\alpha \beta^{+} \mathrm{CD} 3^{+}$ $\mathrm{T}$ cells and $\mathrm{CD}^{+} 8^{+}$cells were the major sources of IL-36 $\alpha$ in minor salivary glands of pSS patients (57). In addition, serum levels of IL-36 $\alpha$ in autoimmune blistering disease patients were significantly higher than those in controls, and elevated IL-36 $\alpha$ was related to disease activity (56) (Table 1).

\section{CONCLUSION}

IL-36 is a member of the IL- 1 superfamily. IL-36 has similarities to IL-1 in its signaling pathway and biochemical function. IL-36 is expressed in a variety of cells and tissues. Keratinocytes are important target cells for IL-36. In recent years, growing evidence has indicated that IL-36 plays important roles in systemic inflammatory diseases. Both animal and in vitro experimental studies and patient samples showed the effect of IL-36 in the pathogenesis of these disorders. However, several points should be noted. Mutations in IL-36RN can lead to DITRA, which is correlated with a severe course of pustular psoriasis. The IL-23/IL-17/IL-22 axis, which is induced by IL-36, plays an important role in psoriasis. SLE is a complex autoimmune disease with a poor prognosis. A previous study suggested that the pathogenesis of SLE correlated with abnormal B-cell frequency or function (58). The relationship between IL-36 and B cells is rarely discussed. Therefore, how IL-36 is involved in the pathogenesis of SLE by regulating the dysfunction of B cells warrants further

\section{REFERENCES}

1. Dinarello C, Arend W, Sims J, Smith D, Blumberg H, O’Neill L, et al. IL-1 family nomenclature. Nat Immunol. (2010) 11:973. doi: 10.1038/ni1110-973

2. Gabay C, Towne JE. Regulation and function of interleukin-36 cytokines in homeostasis and pathological conditions. J Leukoc Biol. (2015) 97:645-52. doi: 10.1189/jlb.3RI1014-495R

3. Yang J, Meyer M, Muller AK, Bohm F, Grose R, Dauwalder $\mathrm{T}$, et al. Fibroblast growth factor receptors 1 and 2 in keratinocytes control the epidermal barrier and cutaneous homeostasis. J Cell Biol. (2010) 188:935-52. doi: 10.1083/jcb.2009 10126

4. Bassoy EY, Towne JE, Gabay C. Regulation and function of interleukin-36 cytokines. Immunol Rev. (2018) 281:169-78. doi: 10.1111/imr.12610

5. Clancy DM, Henry CM, Sullivan GP, Martin SJ. Neutrophil extracellular traps can serve as platforms for processing and activation of IL-1 family cytokines. FEBS J. (2017) 284:1712-25. doi: 10.1111/febs.14075

6. Henry CM, Sullivan GP, Clancy DM, Afonina IS, Kulms D, Martin SJ. Neutrophil-derived proteases escalate inflammation through activation of IL-36 family cytokines. Cell Rep. (2016) 14:708-22. doi: 10.1016/j.celrep.2015.12.072

7. Macleod T, Doble R, McGonagle D, Wasson CW, Alase A, Stacey $\mathrm{M}$, et al. Neutrophil Elastase-mediated proteolysis activates the antiinflammatory cytokine IL-36 Receptor antagonist. Sci Rep. (2016) 6:24880. doi: $10.1038 /$ srep 24880

8. Ainscough JS, Macleod T, McGonagle D, Brakefield R, Baron JM, Alase $A$, et al. Cathepsin $S$ is the major activator of the psoriasis-associated

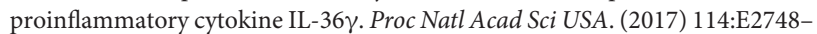
57. doi: 10.1073/pnas.1620954114

9. Cavalli G, Dinarello CA. Suppression of inflammation and acquired immunity by IL-37. Immunol Rev. (2018) 281:179-90. doi: 10.1111/imr.12605

10. Cavalli G, Dinarello CA. Anakinra therapy for non-cancer inflammatory diseases. Front Pharmacol. (2018) 9:1157. doi: 10.3389/fphar.2018.01157 investigation. Since the above findings indicate a significant relationship between IL-36 and disease activity in different inflammatory autoimmune diseases, including SLE, there is great potential for IL-36 as a biomarker for these diseases. To test the potential of IL-36 as a disease activity marker, a specific cohort of patients with preclinical disease, early disease, or long-standing disease, which are situations in which IL-36 has demonstrated diagnostic or predictive utility, should be recruited.

In conclusion, much progress has been made in recent years in studying the role of IL-36 cytokines in the pathogenesis of systemic inflammatory diseases, especially regarding their roles in regulating skin inflammation. The information in this review may aid in guiding the development of novel targeted therapeutic strategies.

\section{AUTHOR CONTRIBUTIONS}

Z-CY, X-YaL, X-YoL, W-DX, A-FH, and L-CS designed and wrote this paper. All of the co-authors agreed on the submission of the paper.

\section{FUNDING}

This work was supported by grants from the National Natural Science Foundation of China (81701606) and the Sichuan Provincial Science and Technology Program (2019YJ0540).
11. Foster AM, Baliwag J, Chen CS, Guzman AM, Stoll SW, Gudjonsson JE, et al. IL-36 promotes myeloid cell infiltration, activation, and inflammatory activity in skin. J Immunol. (2014) 192:6053-61. doi: 10.4049/jimmunol.1301481

12. Carriere V, Roussel L, Ortega N, Lacorre DA, Americh L, Aguilar L, et al. IL-33, the IL-1-like cytokine ligand for ST2 receptor, is a chromatinassociated nuclear factor in vivo. Proc Natl Acad Sci USA. (2007) 104:282-7. doi: 10.1073/pnas.0606854104

13. Carrier Y, Ma HL, Ramon HE, Napierata L, Small C, O’Toole M, et al. Interregulation of Th17 cytokines and the IL-36 cytokines in vitro and in vivo: implications in psoriasis pathogenesis. J Invest Dermatol. (2011) 131:2428-37. doi: 10.1038/jid.2011.234

14. Mahil SK, Catapano M, Di Meglio P, Dand N, Ahlfors H, Carr IM, et al. An analysis of IL-36 signature genes and individuals with IL1RL2 knockout mutations validates IL-36 as a psoriasis therapeutic target. Sci. Transl. Med. 9:eaan2514. doi: 10.1126/scitranslmed.aan2514

15. Swindell WR, Beamer MA, Sarkar MK, Loftus S, Fullmer J, Xing X, et al. RNA-seq analysis of IL-1B and IL-36 responses in epidermal keratinocytes identifies a shared MyD88-dependent gene signature. Front Immunol. (2018) 9:80. doi: 10.3389/fimmu.2018.00080

16. Muller A, Hennig A, Lorscheid S, Grondona P, Schulze-Osthoff K, Hailfinger $\mathrm{S}$, et al. IkappaBzeta is a key transcriptional regulator of IL-36-driven psoriasis-related gene expression in keratinocytes. Proc Natl Acad Sci USA. (2018) 115:10088-93. doi: 10.1073/pnas.1801377115

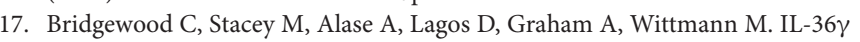
has proinflammatory effects on human endothelial cells. Exp Dermatol. (2017) 26:402-8. doi: 10.1111/exd.13228

18. Vigne S, Palmer G, Lamacchia C, Martin P, Talabot-Ayer D, Rodriguez E, et al. IL-36R ligands are potent regulators of dendritic and T cells. Blood. (2011) 118:5813-23. doi: 10.1182/blood-2011-05-356873

19. Dietrich D, Martin P, Flacher V, Sun Y, Jarrossay D, Brembilla N, et al. Interleukin-36 potently stimulates human M2 macrophages, Langerhans cells and keratinocytes to produce pro-inflammatory cytokines. Cytokine. (2016) 84:88-98. doi: 10.1016/j.cyto.2016.05.012 
20. Milora KA, Fu H, Dubaz O, Jensen LE. Unprocessed interleukin-36 $\alpha$ regulates psoriasis-like skin inflammation in cooperation with interleukin-1. J Invest Dermatol. (2015) 135:2992-3000. doi: 10.1038/jid.2015.289

21. Harusato A, Abo H, Ngo VL, Yi SW, Mitsutake K, Osuka S, et al. IL$36 \gamma$ signaling controls the induced regulatory $\mathrm{T}$ cell-Th9 cell balance via NFkB activation and STAT transcription factors. Mucosal Immunol. (2017) 10:1455-67. doi: 10.1038/mi.2017.21

22. Russell SE, Horan RM, Stefanska AM, Carey A, Leon G, Aguilera $\mathrm{M}$, et al. IL-36 $\alpha$ expression is elevated in ulcerative colitis and promotes colonic inflammation. Mucosal Immunol. (2016) 9:1193-204. doi: $10.1038 / \mathrm{mi} .2015 .134$

23. Mutamba S, Allison A, Mahida Y, Barrow P, Foster N. Expression of IL1 Rrp2 by human myelomonocytic cells is unique to DCs and facilitates DC maturation by IL-1F8 and IL-1F(2017) 9. Eur J Immunol. (2012) 42:607-17. doi: 10.1002/eji.201142035

24. Mahil SK, Twelves S, Farkas K, Setta-Kaffetzi N, Burden AD, Gach JE, et al. AP1S3 Mutations cause skin autoinflammation by disrupting keratinocyte autophagy and up-regulating IL-36 production. J Invest Dermatol. (2016) 136:2251-9. doi: 10.1016/j.jid.2016.06.618

25. Lian LH, Milora KA, Manupipatpong KK, Jensen LE. The doublestranded RNA analogue polyinosinic-polycytidylic acid induces keratinocyte pyroptosis and release of IL-36 $\gamma$. J Invest Dermatol. (2012) 132:1346-53. doi: $10.1038 /$ jid.2011.482

26. van de Veerdonk FL, Stoeckman AK, Wu G, Boeckermann AN, Azam T, Netea MG, et al. IL-38 binds to the IL-36 receptor and has biological effects on immune cells similar to IL-36 receptor antagonist. Proc Natl Acad Sci USA. (2012) 109:3001-5. doi: 10.1073/pnas.1121534109

27. Ichii O, Otsuka S, Sasaki N, Yabuki A, Ohta $H$, Takiguchi $M$, et al. Local overexpression of interleukin-1 family, member 6 relates to the development of tubulointerstitial lesions. Lab Invest. (2010) 90:459-75. doi: 10.1038/labinvest.2009.148

28. Chu M, Wong CK, Cai Z, Dong J, Jiao D, Kam NW, et al. Elevated expression and pro-inflammatory activity of IL-36 in patients with systemic lupus erythematosus. Molecules. (2015) 20:19588-604. doi: 10.3390/molecules201019588

29. Ismail SM, Abd EMK, Mohamed MS. Serum levels of pentraxin3 and interlukin36 in patients with systemic lupus and their relation to disease activity. Egypt J Immunol. (2018) 25:81-91.

30. Mai SZ, Li CJ, Xie XY, Xiong H, Xu M, Zeng FQ, et al. Increased serum IL-36 $\alpha$ and IL-36 $\gamma$ levels in patients with systemic lupus erythematosus: association with disease activity and arthritis. Int Immunopharmacol. (2018) 58:103-8. doi: 10.1016/j.intimp.2018.03.011

31. Xu WD, Su LC, Xie QB, Zhao Y, Liu Y. Interleukin-2-inducible T-cell kinase expression and relation to disease severity in systemic lupus erythematosus. Clin Chim Acta. (2016) 463:11-7. doi: 10.1016/j.cca.2016.10.010

32. Pan HF, Leng RX, Feng CC, Li XP, Chen GM, Li BZ, et al. Expression profiles of Th17 pathway related genes in human systemic lupus erythematosus. $\mathrm{Mol}$ Biol Rep. (2013) 40:391-9. doi: 10.1007/s11033-012-2073-2

33. Amarilyo G, Lourenco EV, Shi FD, La Cava A. IL-17 promotes murine lupus. J Immunol. (2014) 193:540-3. doi: 10.4049/jimmunol.1400931

34. Robinson A, Van Voorhees AS, Hsu S, Korman NJ, Lebwohl MG, Bebo BFJr, et al. Treatment of pustular psoriasis: from the Medical Board of the National Psoriasis Foundation. J Am Acad Dermatol. (2012) 67:279-88. doi: 10.1016/j.jaad.2011.01.032

35. Chandran V, Schentag CT, Brockbank JE, Pellett FJ, Shanmugarajah S, Toloza SM, et al. Familial aggregation of psoriatic arthritis. Ann Rheum Dis. (2009) 68:664-7. doi: 10.1136/ard.2008.089367

36. Fan X, Yang S, Huang W, Wang ZM, Sun LD, Liang YH, et al. Fine mapping of the psoriasis susceptibility locus PSORS1 supports HLA-C as the susceptibility gene in the Han Chinese population. PLoS Genet. (2008) 4:e1000038. doi: 10.1371/journal.pgen.1000038

37. Stawczyk-Macieja M, Rebala K, Szczerkowska-Dobosz A, Wysocka J, Cybulska L, Kapinska E, et al. Evaluation of psoriasis genetic risk based on five susceptibility markers in a population from Northern Poland. PLoS ONE. (2016) 11:e0163185. doi: 10.1371/journal.pone.0163185

38. Zuo X, Sun L, Yin X, Gao J, Sheng Y, Xu J, et al. Whole-exome SNP array identifies 15 new susceptibility loci for psoriasis. Nat Commun. (2015) 6:6793. doi: $10.1038 /$ ncomms7793
39. Tauber M, Bal E, Pei XY, Madrange M, Khelil A, Sahel H, et al. IL36RN Mutations affect protein expression and function: a basis for genotypephenotype correlation in pustular diseases. J Invest Dermatol. (2016) 136:1811-9. doi: 10.1016/j.jid.2016.04.038

40. Hospach T, Glowatzki F, Blankenburg F, Conzelmann D, Stirnkorb C, Mullerschon CS, et al. Scoping review of biological treatment of deficiency of interleukin-36 receptor antagonist (DITRA) in children and adolescents. Pediatr Rheumatol Online J. (2019) 17:37. doi: 10.1186/s12969-019-0338-1

41. Sehat M, Talaei R, Dadgostar E, Nikoueinejad H, Akbari H. Evaluating serum levels of IL-33, IL-36, IL-37 and gene expression of IL-37 in patients with psoriasis vulgaris. Iran J Allergy Asthma Immunol. (2018) 17:179-87.

42. He Q, Chen HX, Li W, Wu Y, Chen SJ, Yue Q, et al. IL-36 cytokine expression and its relationship with p38 MAPK and NF$\mathrm{\kappa B}$ pathways in psoriasis vulgaris skin lesions. J Huazhong Univ Sci Technolog Med Sci. (2013) 33:594-9. doi: 10.1007/s11596-01 3-1164-1

43. Bachelez H, Choon SE, Marrakchi S, Burden AD, Tsai TF, Morita A, et al. Inhibition of the interleukin-36 pathway for the treatment of generalized pustular psoriasis. $N$ Engl J Med. (2019) 380:981-3. doi: 10.1056/NEJMc18 11317

44. Ohko K, Nakajima K, Kataoka S, Takaishi M, Sano S. IL-36 signaling is essential for psoriatic inflammation through the augmentation of innate immune responses. J Invest Dermatol. (2018) 139:1400-4. doi: 10.1016/j.jid.2018.12.003

45. Johnston A, Xing X, Wolterink L, Barnes DH, Yin Z, Reingold L, et al. IL-1 and IL-36 are dominant cytokines in generalized pustular psoriasis. J Allergy Clin Immunol. (2017) 140:109-20. doi: 10.1016/j.jaci.2016. 08.056

46. Tortola L, Rosenwald E, Abel B, Blumberg H, Schafer M, Coyle AJ, et al. Psoriasiform dermatitis is driven by IL-36-mediated DC-keratinocyte crosstalk. J Clin Invest. (2012) 122:3965-76. doi: 10.1172/JCI63451

47. Frey S, Derer A, Messbacher ME, Baeten DL, Bugatti S, Montecucco C, et al. The novel cytokine interleukin-36 $\alpha$ is expressed in psoriatic and rheumatoid arthritis synovium. Ann Rheum Dis. (2013) 72:1569-74. doi: 10.1136/annrheumdis-2012-2 02264

48. Lamacchia C, Palmer G, Rodriguez E, Martin P, Vigne S, Seemayer $\mathrm{CA}$, et al. The severity of experimental arthritis is independent of IL36 receptor signaling. Arthritis Res Ther. (2013) 15:R38. doi: 10.118 $6 / \operatorname{ar} 4192$

49. Li T, Chubinskaya S, Esposito A, Jin X, Tagliafierro L, Loeser R, et al. TGF- $\beta$ type 2 receptor-mediated modulation of the IL-36 family can be therapeutically targeted in osteoarthritis. Sci Transl Med. 11:eaan2585. doi: 10.1126/scitranslmed.aan2585

50. Scheibe K, Backert I, Wirtz S, Hueber A, Schett G, Vieth M, et al. IL-36R signalling activates intestinal epithelial cells and fibroblasts and promotes mucosal healing in vivo. Gut. (2017) 66:823-38. doi: 10.1136/gutjnl-2015-3 10374

51. Nishida A, Hidaka $K$, Kanda $T$, Imaeda $H$, Shioya $M$, Inatomi $\mathrm{O}$, et al. Increased expression of interleukin-36, a member of the interleukin-1 cytokine family, in inflammatory bowel disease. Inflamm Bowel Dis. (2016) 22:303-14. doi: 10.1097/MIB.0000000000 000654

52. Rahman P, Sun S, Peddle L, Snelgrove T, Melay W, Greenwood C, et al. Association between the interleukin-1 family gene cluster and psoriatic arthritis. Arthritis Rheum. (2006) 54:2321-5. doi: 10.1002/art. 21928

53. van Tubergen A, Weber U. Diagnosis and classification in spondyloarthritis: identifying a chameleon. Nat Rev Rheumatol. (2012) 8:253-61. doi: $10.1038 /$ nrrheum.2012.33

54. Chou CT, Timms AE, Wei JC, Tsai WC, Wordsworth BP, Brown MA. Replication of association of IL1 gene complex members with ankylosing spondylitis in Taiwanese Chinese. Ann Rheum Dis. (2006) 65:1106-9. doi: 10.1136/ard.2005.046847

55. Guo ZS, Li C, Lin ZM, Huang JX, Wei QJ, Wang XW, et al. Association of IL-1 gene complex members with ankylosing spondylitis in Chinese Han population. Int J Immunogenet. (2010) 37:33-7. doi: 10.1111/j.1744-313X.2009.00889.x 
56. Zebrowska A, Wozniacka A, Juczynska K, Ociepa K, Waszczykowska E, Szymczak I, et al. Correlation between IL36 $\alpha$ and IL17 and activity of the disease in selected autoimmune blistering disease s. Mediators Inflamm. (2017) 2017:8980534. doi: 10.1155/2017/89 80534

57. Ciccia F, Accardo-Palumbo A, Alessandro R, Alessandri C, Priori R, Guggino $\mathrm{G}$, et al. Interleukin-36 $\alpha$ axis is modulated in patients with primary Sjogren's syndrome. Clin Exp Immunol. (2015) 181:230-8. doi: 10.1111/cei.12644

58. Samy E, Wax S, Huard B, Hess H, Schneider P. Targeting BAFF and APRIL in systemic lupus erythematosus and other antibody-associated diseases. Int Rev Immunol. (2017) 36:3-19.doi: 10.1080/08830185.2016.1276903
Conflict of Interest: The authors declare that the research was conducted in the absence of any commercial or financial relationships that could be construed as a potential conflict of interest.

Copyright (C) 2019 Yuan, Xu, Liu, Liu, Huang and Su. This is an open-access article distributed under the terms of the Creative Commons Attribution License (CC BY). The use, distribution or reproduction in other forums is permitted, provided the original author(s) and the copyright owner(s) are credited and that the original publication in this journal is cited, in accordance with accepted academic practice. No use, distribution or reproduction is permitted which does not comply with these terms. 